\title{
Applications of a computer algebra system in introductory statistics
}

\author{
RITA SNYDER \\ Denison University, Granville, Ohio
}

\begin{abstract}
Maple, a computer algebra system, is employed in introductory statistics courses to promote conceptual learning by students of statistical principles without direct use of mathematics. Maple's symbolic computation, graphic display, and animation capabilities support an integrated set of procedures for active study of sampling distributions and concepts related to samples, populations, and statistical decision making, error, and power. Students select parameter values for one-line commands and examine the effects of alternatives on computer-generated graphical representations of distributions. The 20 procedures written to support active student exploration of basic statistical concepts are described, and examples of exercises to support their use are provided.
\end{abstract}

Symbolic computation has been used successfully in undergraduate mathematics courses such as calculus, linear algebra, and advanced probability and statistics (Karian, 1992). This paper describes applications of a computer algebra system, Maple, for designing procedures to support conceptual learning of statistical principles in an introductory statistics course for psychology students. Maple is used to create visualizations of distributions to allow students to explore important aspects of statistical representation without direct use of mathematics.

Teaching introductory statistics to psychology students may be approached in several ways. Most textbooks blend a conceptual presentation with procedural instruction, both of which are needed to appreciate the basis for, and proper application of, statistics (see, e.g., Hiebert \& Lefevre, 1986). However, weak mathematical preparation and anxiety about mathematical material often impede learning for a significant number of students (Lalonde \& Gardner, 1993). Computer-based instruction offers possibilities for active learning that could prove useful for teaching both conceptual and procedural material in the introductory statistics course. For example, statistical computation packages such as SAS and SPSSx allow students to focus on the application and interpretation of statistical tests of realistic databases, and have been used with some success for these purposes (e.g., Snyder, 1994; Walsh, 1994). However, development has been more limited in the area of integrated computer-based approaches for helping mathematically weak students develop a better conceptual understanding of the basis for statistical testing.

Karian (1994; see also Karian \& Tanis, 1995) has described development of more than 130 Maple procedures

This work was supported in part by Grant P116B30079 from the Fund for the Improvement of PostSecondary Education (FIPSE) of the Department of Education. Correspondence may be sent to R. Snyder, Department of Psychology, Denison University, Granville, OH 43023 (e-mail: snyder@cc.denison.edu). for use in an advanced probability and statistics course. These procedures support, among other activities, the generation of random samples from a variety of discrete and continuous distributions (including sampling distributions) and the plotting of sampled data as well as empirical and theoretical distributions. I have designed 20 Maple procedures that incorporate some of Karian's set for use by introductory statistics students. Each procedure, which is executed with a one-line command, completes a series of steps and (with one exception) generates color graphical representations of distributions for study of key statistical concepts and principles.' For each procedure, students must select parameter values and then interpret the changing effects of alternatives they explore.

These procedures focus on three important areas for understanding the bases of statistical analysis: (1) relationships among samples, populations, and sampling distributions; (2) relationships among sampling distributions; and (3) statistical power. Statistical concepts that may be explored with these Maple procedures, as well as examples of exercises used in an introductory statistics class, are described in the next sections.

\section{Relationships Among Samples, Populations, and Sampling Distributions}

A set of 7 procedures supports examination of these relationships for user-specified populations. To execute each procedure, the student enters values for the population mean ( $\mathrm{mu}$ ) and standard deviation ( $S D$; sigma), as well as for the sample sizes $(N s)$ drawn at random from that population. In some cases, the student may also control the number of random samples on which representations of populations and sampling distributions are based. This set of procedures consists of the following:

1. est (mu, sigma, smallN, bigN, Nsamples). This displays values of means and $S D$ s for a number of small and large samples drawn randomly from a normally distrib- 
uted population of scores. The student selects values for population mean and $S D$, small and large sample sizes, and the number of samples he or she wishes to draw. Sample statistics, as well as means and $S D$ s of the sample statistics, are shown. This procedure helps students explore parameter estimation and introduces them to the examination of sets of sample statistics.

2. drawsdistn (mu, sigma, $N$ ). This displays a population distribution (normal) and the sampling distribution of means of samples of size $N$ drawn randomly from that population. The student selects values for the population mean and $S D$ and for sample $N \mathrm{~s}$. This procedure may be used in exercises to examine relationships between population and sampling distributions as well as among population $S D$, sample size, and standard error $(S E)$.

3. animsdistn (mu, sigma). This animates the change in $S E$ as random samples from a normal distribution increase and decrease in size. The population distribution is presented as a static display against which the changes in $S E$ for the sampling distributions representing samples of increasing or decreasing $N \mathrm{~s}$ are observed. This is especially useful for demonstrations and class discussion.

4. drawsample1 (sigma, mu, smallN, bigN, Nsamples). This displays histograms of sample means based on a specified number of small and large samples drawn randomly from a normal distribution. The student selects population parameter values, sizes of small and large sample $N$ s, and the number of samples he or she wishes to draw from the population. This procedure may be used as part of a set of exercises concerning the central limit theorem.

5. drawsample2 (df, smallN, bigN, Nsamples).This is analogous to drawsample1, but samples from a skewed (chi-square) distribution. This is also used as part of a set of exercises about the central limit theorem.

6. drawsamplelt (sigma, mu, smallN, bigN, Nsamples).

7. drawsample2t (df; smallN, bigN, Nsamples).

These last two procedures (6 and 7) are variations of drawsample1 and drawsample2 that impose a normal distribution over each histogram to represent a theoretical sampling distribution. They are used to illustrate the connection between samples drawn from specified populations and their representation by sampling distributions, and they, too, reinforce earlier conceptualizations of the central limit theorem.

An illustration of how these procedures may be used by students to explore relationships between population distributions and sampling distributions is provided by the following exercise. Students are asked to use the procedure drawsdistn to create superimposed graphs of a population of IQ scores and the sampling distribution that results when random samples of a selected size are drawn from that population. Students specify a mean of 100 and an $S D$ of 15 for each execution of the procedure, but change the sample size as directed by the questions assigned for this exercise. Samples of the Maplegenerated output of two executions of this procedure for sample sizes of $N=6$ and $N=30$ are shown in Figure 1.

The students examine these figures and answer the following assigned questions to articulate the similarities and differences between these pairs of distributions:

1. Select a small sample size (this is your value for $N$ ). How does the sampling distribution for samples of this size compare to the population distribution? Why are they different?

2. Select a larger $N$. How does this affect the comparison between the two types of distributions? Explain why the comparison does or does not change.

3. Looking at the two sets of distributions, describe the effect of changing the sample size on the mean and $S E$ of the sampling distribution. Select one or two new values for $N$ to test your generalization.

4. Can the value of the $S E$ of the sampling distribution of the mean be larger than the population $S D$ ? Explain.

5. Assume you randomly sampled from the given population and obtained a sample with mean $=105$. Would you be more likely to obtain this sample mean when $N=6$ or when $N=30$ ? Explain.
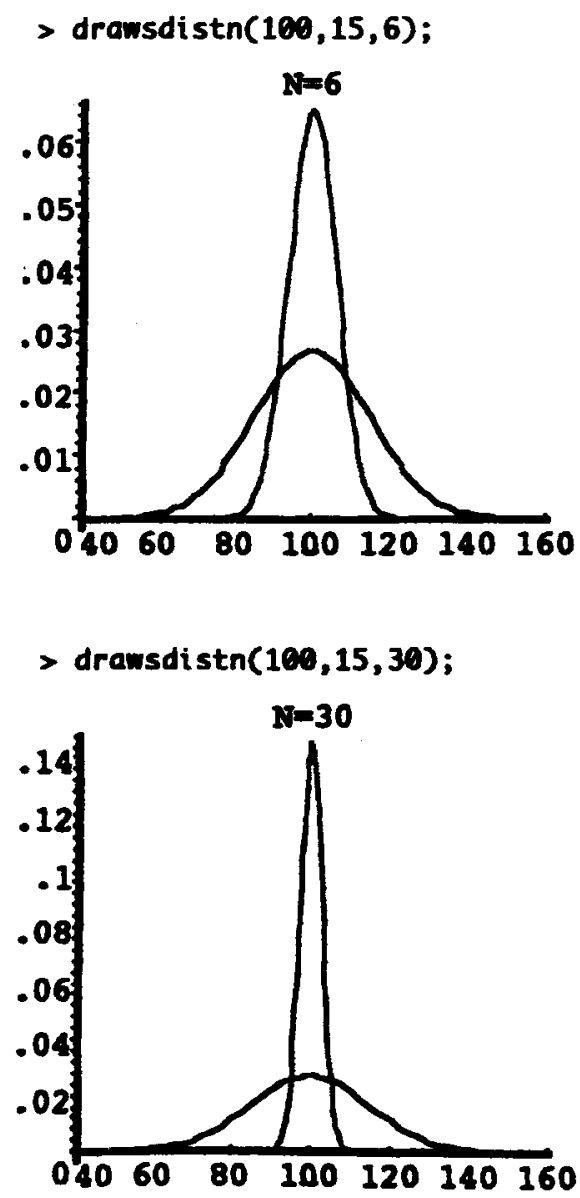

Figure 1. Comparison of population distribution and sampling distribution based on random samples from the population, for $N=6($ top) and $N=30$ (bottom). 
In answering questions such as these, students see that the means and shapes of the population and sampling distributions remain the same, but that the variability of the sampling distribution is affected by sample size. They are able to apply concepts articulated in their text about the relationship between $S E$ and population $S D$ (Question 4), as well as to explore the impact of sample size on the magnitude of error that may result when samples are used to provide population estimates (Question 5).

Since random sampling is reinitiated with each execution of a procedure, the students also directly observe sampling variations from the plots they generate. This has distinct advantages over static drawings provided by textbooks in terms of reinforcing the importance of using statistical techniques to deal with sampling error. The animated display of a sampling distribution (Procedure animsdistn) also may be used to illustrate a changing $S E$ as $N$ changes, and is shown against the static population distribution from which the samples were drawn. This procedure creates a dynamic display to help students visualize relationships between population $S D$, sample size, and $S E$, and is especially helpful for stimulating class discussion of these concepts. Finally, the procedures included in this set may be used to examine (1) how sample statistics compare with population parameters (numerical output); (2) the effects of sample size on sampling distribution characteristics (e.g., histogram plots with optional imposed normal distribution may be used to examine shape and dispersion); and (3) how sampling distributions of sample means randomly selected from both normal and skewed distributions approximate a normal distribution when large samples are drawn (to illustrate the central limit theorem).

\section{Relationships Among Sampling Distributions}

A set of 9 Maple procedures allows students to explore relationships among commonly used sampling distributions. The shape and dispersion of $t, F$, and chi-square distributions produced by student-selected degrees of freedom ( $d f)$ may be examined in isolation or contrasted against a unit normal distribution. Animated displays may also be used to observe the changing shape of each distribution with changing $d f$. Each animated distribution is displayed against a unit normal distribution. This set of procedures is as follows:(1) $\operatorname{drawt}(\mathrm{df})$; (2) drawf (dfnum, dfdenom); (3) drawchi(df); (4) drawtn (df); (5) drawfn(dfnum, dfdenom); (6) drawchin(df); (7) animt ( ); (8) animf ( ); (9) animchi( ). (1), (2), and (3) each allow the student to select values for $d f$ and display the resultant sampling distributions $(t, F$, and chi square, respectively). The student may observe the relationship between $d f$ and the shapes of the distributions. (4), (5), and (6) add a unit normal sampling distribution to plots of $t, F$, and chi-square distributions, respectively, with student-selected $d f$. The student may observe the approximation to the normal distribution with increasing $d f$. (7), (8), and (9) animate the change in the shape and dispersion of $t, F$, and chi-square distributions, respec- tively, with changing $d f$. A static unit normal distribution is displayed, against which the changing $t, F$, or chisquare distributions are observed. These programs are especially useful for demonstrations and class discussion.

An illustration of how these procedures may be used by students to explore relationships between $t$ and normal distributions is provided by the following exercise. Students are asked to use the procedures drawt and drawtn to generate graphical displays of $t$ distributions alone, as well as superimposed $t$ and unit normal distributions. Figure 2 shows an example of two graphs produced by executing drawtn for $d f=2$ and $d f=12$, respectively. The unit normal distribution is shown in black on the computer screen, and the $t$ distribution is shown in red.

The students are asked to generate and examine distributions with questions such as the following:

1. Generate three $t$ distributions with the procedure drawt(df), using small, moderate, and large values for $\mathrm{df}$, respectively. Label each graph with the $d f$ used to produce it. How do the three distributions compare in terms of (a) their means; (b) their $S E \mathrm{~s}$; and (c) their shapes?
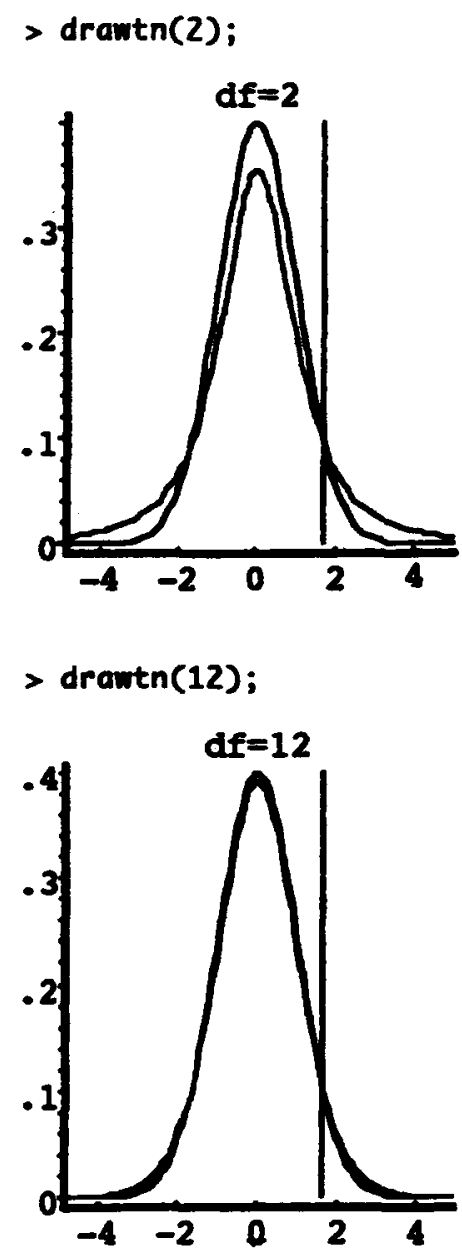

Figure 2. Plots of superimposed $t$ and unit normal sampling distributions when $d f=2$ (top) and 12 (bottom). 
2. Repeat Question 1, but use the procedure $\operatorname{drawtn}(\mathrm{df})$ instead. The decision criterion for alpha $=.05$ on the normal distribution is shown with a vertical line. Describe how the two distributions compare in terms of means, $S E \mathrm{~s}$, and shapes for small, moderate, and large $d f \mathrm{~s}$.

3. Approximate the location of the decision criterion for alpha $=.05$ on the $t$ distribution with the small $d f$ that you created for Question 2. Should it be located above or below the decision criterion shown on the normal distribution? Explain.

4. How does the relative proportion of samples for which the null hypothesis is rejected compare for the normal and $t$ distributions when the sample is small? How do the values of sample means for which the null hypothesis is rejected compare? Explain.

5 . Why should a $t$ distribution, rather than a normal distribution, be used for statistical testing when the population $S D$ must be estimated from a sample?

In answering questions such as these, the students focus on the characteristics of each sampling distribution, especially in relation to a normal distribution (Questions 1 and 2). In addition, they learn the importance of employing the appropriate sampling distribution for the type of design and data characteristics they obtain from a given study (Questions 3, 4, and 5). Concepts introduced earlier in the course related to sampling error and the central limit theorem are also reinforced by producing displays of various sampling distributions and examining their respective characteristics.

\section{Statistical Power}

Four procedures are available for use in exercises which allow students to examine how the power of a statistical test is affected by four factors: the effect size (d); the sample size $(N)$; the population $S D$ (sigma); and the Type I error probability (alpha). One procedure allows the students to designate sets of values for effect size, sample size, population $S D$, and Type I error to compare graphs of the resultant "true" and null hypothesis sampling distributions. A vertical line representing the decision criterion is also shown. The three animated procedures are included to provide dynamic displays for examining relationships between statistical power and effect size, sample size, and Type I error:

1. powergen ( $N$, sigma, d, alpha) displays pairs of distributions, one representing the sampling distribution under the null hypothesis, and the other representing the "true" population sampling distribution. A vertical line is drawn to represent the alpha decision criterion value on the null distribution. The student may complete a series of exercises designed to explore the impact of population $S D$, sample size, effect size, and alpha level on the power of a statistical test. This procedure may also be used to illustrate important concepts such as the relationship between Type I and Type II error.

2. powerd ( $N$, sigma, alpha) animates the result of changes in effect size by increasing and decreasing the distance between the means of the null hypothesis and "true" sampling distributions. Values of $N$, sigma, and alpha are user defined and held constant. The student observes the changing proportion of the "true" sampling distribution for which the null hypothesis would be rejected. This procedure is useful for demonstrations and class discussion.

3. poweralpha $(N$, sigma, d) animates a changing alpha level by moving a vertical line denoting the decision criterion value on the null sampling distribution. Values of $N$, sigma, and effect size are user defined and held constant. The student may observe the changes in the proportions of both the null and "true" sampling distributions for which the null hypothesis would be rejected as the alpha level changes. This procedure is useful for demonstrations and class discussion.

4. powern (sigma, d, alpha) animates the effect of changing sample size by changing the $S E$ of both the null and the "true" sampling distributions. Values of sigma, effect size, and alpha are user defined and held constant. Students observe the changing proportion of the "true" distribution for which the null hypothesis would be rejected as $N$ changes. This procedure is useful for demonstrations and class discussion.

An illustration of how these procedures may be used by students to explore the relationship between Type I and Type II errors is provided by the following example. Students execute powergen using different values for alpha and holding sample size, sigma, and effect size constant. Figure 3 shows the pair of distributions produced for alpha values of .05 and .01 . This pair of normal distributions represents the sampling distributions generated by the null hypothesis (on the left) and the "true" sampling distribution for a medium-effect size. The decision criterion drawn on the null distribution is only shown for the upper tail, indicating the rejection region for a one-tail test.

Questions such as the following help students identify how Type I and Type II errors are represented by these sampling distributions as well as how they are related:

1. For a one-tailed test, set alpha $=.05$ and graph the two distributions. Label the graph with your alpha value. On the output, color the region of the graph that shows Type I error. Use a different color (or pattern) to mark the region showing Type II error.

\section{Repeat Question 1, but set alpha $=.01$.}

3. Describe the relationship between Type I and Type II errors illustrated by comparing the colored regions for Questions 1 and 2.

4. If you wanted to compare the regions indicating Type I and Type II errors for a two-tailed test with alpha = .05 , what value would you set for alpha in this procedure? Explain.

5. Repeat Question 1, using your new value for alpha. Label the graph with your alpha value for this run. Describe how Type II error was affected when you switched from a one-tailed test to a two-tailed test.

The following questions may be added to help connect these concepts to statistical power: 
$>$ powergen $(24,10, .5, .05)$;

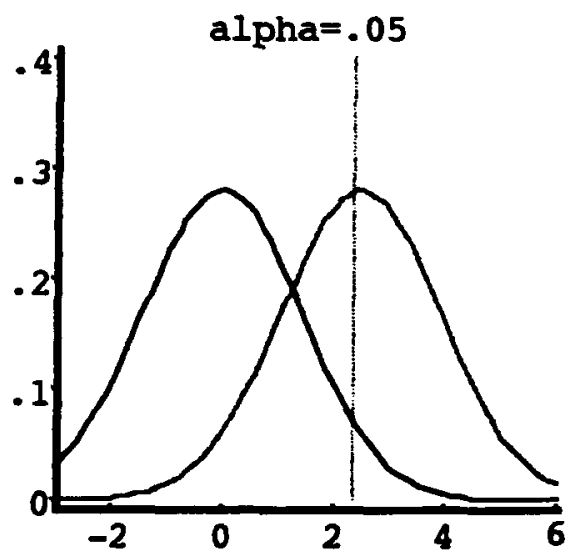

$>$ powergen $(24,10, .5, .01)$;

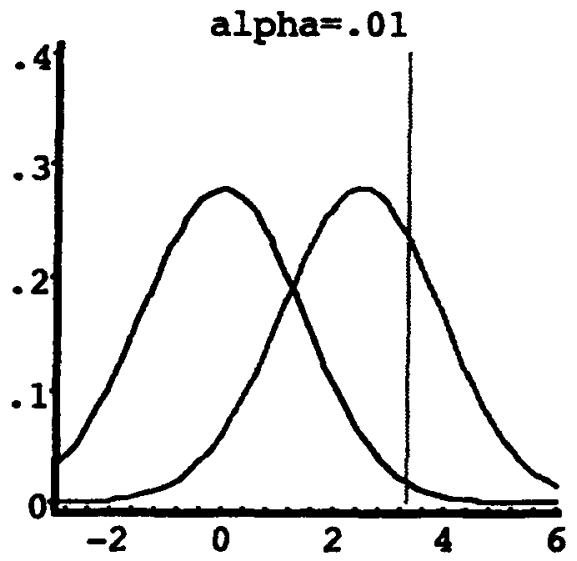

Figure 3. Representation of null hypothesis and "true" sampling distributions for alpha $=.05$ (top) and alpha $=.01$ (bottom).

6. Since power $=(1-$ Type II error probability $)$, describe the effect on power of decreasing the Type I error probability.

7. Describe the effect on power of using a two-tailed test rather than a one-tailed test.

Sets of questions similar to these may be designed to guide the students' exploration of the impact of changing effect size and changing sample size on the power of statistical tests. Understanding these relationships helps lay the foundation for meaningful discussions of relationships between methodological and statistical aspects of research, as well as of some limitations on the use of statistical testing.

\section{Conclusion}

An understanding of sampling distributions is essential for understanding the basis for statistical analysis.
This set of Maple procedures may be used throughout the semester in an introductory statistics course to represent graphically a variety of characteristics of sampling distributions and basic concepts related to samples, populations, statistical decision making, and statistical error. Because symbolic computation is completed relatively quickly and easily by these Maplebased procedures, students are able to create dynamic learning experiences for themselves that are primarily pictorial, but that illustrate key statistical principles. Since students must select parameter values for each procedure and interpret the effects that their selections have on the graphical displays, they actively explore statistical concepts without mathematical manipulation. By designing appropriate exercises in which these procedures are employed, conceptual learning is enhanced. These types of computer-based procedures afford considerable opportunity for development of different types of exercises for use by introductory statistics students. The versatility of Maple for computation, graphic display, and animation permits development of an integrated set of laboratory exercises that promise to be very useful for supporting conceptual understanding of statistical principles by introductory statistics students.

\section{REFERENCES}

Hiebert, J., \& Lefevre, P. (1986). Conceptual and procedural knowledge in mathematics: An introductory analysis. In J. Hiebert (Ed.), Conceptual and pracedural knowledge: The case of mathematics (pp. 1-28). Hillsdale, NJ: Erlbaum.

KARIAN, Z. A. (Ed.) (1992). Symbolic computation in undergraduate mathematics education (MAA Notes 24). Washington, DC: Mathematical Association of America.

KARIAN, Z. A. (1994, July). Computer algebra systems in statistics. Paper presented at the Fourth International Conference on Teaching Statistics, Marrakech, Morocco.

Karian, Z. A., \& Tanis, E. A. (1995). Probability and statistics explorations with Maple. Englewood Cliffs, NJ: Prentice-Hall.

LALONDE, R. N., \& GaRdNER, R. C. (1993). Statistics as a second language? A model for predicting performance in psychology students. Canadian Journal of Behavioural Science, 25, 108-125.

SNYDER, R. (1994, July). A computer laboratory for teaching statistical reasoning. Paper presented at the American Psychological Society Institute on the Teaching of Psychology, Washington, DC.

WALSH, J. F. (1994). One-way between subjects design: Simulated data and analysis using SAS. Teaching of Psychology, 21, 53-55.

\section{NOTE}

1. These procedures run with both Macintosh and DOS versions of Maple. Contact Waterloo Maple Software at info@maplesoft.on.ca, or at (800) $267-6583$, for specific information about computer requirements for each version. Upon request, additional software needed to run the statistical procedures described in this paper will be provided by the author on either a Macintosh or a DOS diskette.
(Manuscript received November 22, 1994; revision accepted for publication January 27, 1995.) 